\title{
Suchtspezifische Studiengänge an deutschen Hochschulen
}

\author{
M. Klein
}

\author{
Postgraduate Study Programs in the Addiction Field at German Universities
}

\section{Zusammenfassung}

Die von der Deutschen Hauptstelle für Suchtfragen (DHS) im April 2005 veröffentlichten „Empfehlungen zu postgradualen Curricula in der Suchthilfe" werden vorgestellt und einer kritischen Analyse unterzogen. Dies geschieht vor dem Hintergrund langjähriger Forschungs-, Praxis- und Lehrerfahrung des Autors und auf der Basis der aktuellen Diskussionen um Modernisierung und Innovationen in der Suchthilfe. Folgende Hauptkritikpunkte werden herausgearbeitet: Die Empfehlungen stellen erstens keinen ausreichenden Bezug zu Wissenschaft und Forschung in der Suchthilfe her, sind zweitens eher berufsständischer und berufspolitischer Natur, bezogen einseitig auf die Disziplin „Soziale Arbeit“, und orientieren sich drittens an einem konventionellen, restriktiven Verständnis der Suchthilfe und sind dementsprechend als Versuch zur Wahrung bestehender Strukturen und Interessen zu entlarven. Insgesamt ergibt sich, dass die Empfehlungen keinesfalls zur Analyse postgradualer Curricula in der Suchthilfe tauglich sind und daher keinen dementsprechenden Stellenwert erhalten sollten. Vielmehr sind die deutschen Hochschulen, auch in Kommunikation mit innovativen Vertretern der Suchthilfepraxis und der Weiterbildungsträger, aufgefordert, zukunftsfähige Konzepte unter Vermeidung der im Detail elaborierten Kritikpunkte zu erarbeiten bzw. fortzuschreiben. Suchthilfespezifische Studiengänge an deutschen Hochschulen machen nur Sinn, wenn es gelingt, einerseits eine durchgehende intensive Wissenschaftsorientierung sicherzustellen und andererseits einen engen Forschungs-PraxisTransfer und vice versa zu realisieren.

Schlïisselwörter

Suchthilfe $\cdot$ Suchtforschung $\cdot$ Weiterbildung $\cdot$ Studium

\section{Abstract}

In April 2005, the "Deutsche Hauptstelle für Suchtfragen" (DHS) (German office for addiction issues) published recommendations for postgraduate curricula concerning addiction support ("Empfehlungen zu postgradualen Curricula in der Suchthilfe"). These recommendations will be presented and critically analysed. The author relies on many years of experience in research, practice and lecturing, and this presentation is based upon the current discussion related to modern and innovative approaches in addiction support. Main points of criticism are, firstly, that the recommendations are not sufficiently related to science and research in the field of addiction, secondly, that they are rather related to issues of profession and policy with a bias towards "social work", and, thirdly, that they are orientated towards a conventional, restrictive interpretation of addiction support and should, accordingly, be exposed as an attempt to safeguard existing sinecures and interests. As a result, the recommendations are in no way suitable for the analysis of postgraduate curricula concerning addiction support and should, therefore, not be attributed undue importance. German universities should rather communicate with representatives of innovative practice in addiction support and those responsible for further education, in order to develop concepts for the future that avoid the critical points described hereafter. Addiction related courses of study at German universities only make sense, if we succeed, on the one hand, to assure a continuous intensive orientation towards science and, on the other hand, to realise a close transfer from research to practice and back.

Key words

Addiction care · addiction research · continuous training · study

Prof. Dr. Michael Klein · Katholische Fachhochschule Nordrhein-Westfalen,

Kompetenzplattform Suchtforschung · Wörthstraße 10 · 50668 Köln · E-mail: Mikle@kfhnw.de

Bibliografie

Suchttherapie 2005; 6: 192 - 199 @ Georg Thieme Verlag KG Stuttgart · New York

DOI 10.1055/s-2005-858637

ISSN 1439-9903 
Dass suchtspezifische Studiengänge an deutschen Hochschulen angeboten werden, ist eine neue Entwicklung in der Hochschullandschaft, die durch die Einführung von Bachelor- und Masterstudiengängen in der Folge der Novellierung des Hochschulrahmengesetzes von 1997 erst ermöglicht wurde. Dies passt zum größeren Rahmen des so genannten Bologna-Prozesses, der die Angleichung der Studiengänge und -abschlüsse in den EU-Staaten bis zum Jahre 2010 vorsieht. Nachdem bereits vor einigen Jahren die Einrichtung von Lehrstühlen für Suchtforschung an deutschen Universitäten beschlossen und dies inzwischen in Heidelberg/Mannheim und Essen im Bereich der Suchtmedizin auch erfolgreich umgesetzt wurde, ist die hochspezialisierte Aus- und Weiterbildung in suchtspezifischen Themen angesichts der gesundheitspolitischen und -ökonomischen Bedeutung der Suchtstörungen in Deutschland ein logischer Folgeschritt. In Großbritannien, wo hochspezialisierte Universitätsstudiengänge eine längere Tradition als in Deutschland haben, ist von derzeit mindestens 15 suchtspezifischen Studiengängen auf MasterEbene mit sehr unterschiedlichen Schwerpunkten (z. B. Drogentherapie, Suchtforschung, Suchtprävention) auszugehen.

Im Jahre 2001 begann an der Katholischen Fachhochschule Nordrhein-Westfalen (KFH NW) in Köln der bundesweit erste derartige Studiengang. Es handelt sich um den postgradualen Masterstudiengang „Suchthilfe“, der berufsbegleitend in vier Semestern zum akademischen Grad eines „Master of Science in Addiction Prevention and Treatment“ führt. Dieser Studiengang umfasst nach der Akkreditierung 1800 Stunden in einem Weiterbildungsstudium, davon 856 Stunden Präsenzstudium, und bietet derzeit die Wahl zwischen zwei Studienschwerpunkten, Suchtprävention oder Suchttherapie. Die Studierenden müssen sich für einen der beiden Schwerpunkte entscheiden und belegen diesen mit 128 Stunden Schwerpunktstudium, während der nicht gewählte Schwerpunkt mit 64 Stunden studiert wird. Für den Fall, dass der Schwerpunkt „Suchttherapie“ gewählt wird, umfasst das Studium auch eine kognitiv-verhaltenstherapeutische Ausbildung („Suchttherapeut/-in KFH NW), die vom Verband Deutscher Rentenversicherungsträger seit dem Jahr 2000 akkreditiert ist.

Weitere wählbare Schwerpunkte (z.B. Management von Suchthilfeeinrichtungen, angewandte Suchtforschung) sind für die nähere Zukunft geplant. Bisher wurden fünf Studiengänge im jährlichen Abstand mit jeweils 24 Studierenden begonnen bzw. durchgeführt. Inzwischen liegen also Erfahrungen in der Durchführung und Absolvierung des Studiengangs mit mehr als 120 Studierenden vor.

Im April 2005 veröffentlichte die Deutsche Hauptstelle für Suchtfragen (DHS) auf ihrer Website „Empfehlungen zu postgradualen Curricula in der Suchhilfe“ [1]. Diese wurden vom Fachausschuss „Aus-, Fort- und Weiterbildung“ erstellt. Als Autorinnen und Autoren werden Hedi Boss, Irene Helas, Jost Leune, Christina Meyer, Doris Sarrazin und Christoph Simon genannt. Im Folgenden wird dieses Papier vor dem Hintergrund der eigenen langjährigen Erfahrungen mit dem Masterstudiengang Suchthilfe diskutiert. Die im Folgenden gewählte Gliederung orientiert sich zur besseren Verständlichkeit und Nachvollziehbarkeit der Ausführungen an der Gliederung des DHS-Papiers.
In einem einführenden Kapitel „Präambel“ werden die Verdienste der Verbände der Suchtkrankenhilfe um die Qualifizierung der im Bereich der Suchthilfe tätigen Berufsgruppen gewürdigt. Die historisch wichtige Rolle der deutschen Rentenversicherungen (vor allem des bisherigen Dachverbands VDR mit seiner Projektgruppe Sucht), die mit ihrer Empfehlungsvereinbarung „Sucht“ (1978) und der Vereinbarung „Abhängigkeitserkrankungen“ (2001) ganz wesentlich zur Etablierung hochwertiger Qualitätskriterien beigetragen haben, bleibt an dieser und auch an folgenden Stellen unerwähnt. Der Leser kann also den falschen Eindruck gewinnen, dass die DHS - insgesamt und mit ihrem Fachausschuss „Aus-, Fort- und Weiterbildung“ im Speziellen - seit Jahrzehnten der primäre Initiator und Motor für die Qualitätssicherung im Weiterbildungsbereich der Suchthilfe gewesen ist. Was in der Präambel außerdem unerwähnt bleibt, ist die Tatsache, dass postgraduale Curricula, werden sie an Hochschulen angeboten und durchgeführt, stets wissenschaftliches Denken und Handeln fordern und fördern müssen. Stattdessen werden Studiengänge und postgraduale Weiterbildungen unzulässigerweise gleichgesetzt: „Ein Studiengang oder eine postgraduale Weiterbildung zeichnen sich dadurch aus, dass sie aufbauend auf einem grundständigen Ausbildungsabschluss als Sozialarbeiter/-in oder Sozialpädagoge/-in ... weiterführende und spezialisierte Wissensbestände lehren“ (S. 3). Diese Aussagen unterschätzen Bedeutung und Stellenwert wissenschaftlicher Studiengänge, die neben der Vermittlung von Wissen, Methoden und Handlungskompetenzen immer auch die Aufgabe haben, das Bestehende nach wissenschaftlichen Kriterien zu reflektieren und kritisch zu hinterfragen. Die folgenden Ausführungen und kritischen Analysen beziehen sich ausschließlich auf suchtwissenschaftliche Studiengänge und nicht auf suchttherapeutische Weiterbildungen, die überwiegend an privatwirtschaftlich betriebenen Instituten durchgeführt werden.

Angesichts der in der Suchtbehandlungsszene weit verbreiteten und immer wieder beklagten Tendenzen zu Verkrustungen und Mythenbildungen [2-5] ist es vielleicht sogar die wichtigste Aufgabe postgradualer akademischer Curricula für den Bereich der Suchthilfe, zu kritischer Reflexion des beruflichen Handelns anhand wissenschaftlichen Denkens anzuleiten. Ganz offensichtlich nicht in dieser Tradition steht das DHS-Papier: „Diese Empfehlungen gelten im Wesentlichen für die verschiedenen Angebote postgradualer Weiterbildungen, insbesondere verbandlicher Weiterbildungen und wissenschaftlicher Fachhochschul- oder Hochschulstudiengänge“ (S. 3). Aus Sicht der Hochschulen kann diese einseitig verkündete Reichweitendefinition nur als unzulässiger Angriff auf die Freiheit von Lehre und Forschung empfunden und zurückgewiesen werden. Es ist nicht einsehbar und zu legitimieren, dass Verbände, die in der Suchthilfe zweifellos Verdienste erworben haben, einseitig Empfehlungen erlassen, die sich im Geltungsbereich auch auf Hochschulen beziehen, ohne mit diesen abgestimmt zu sein. Bereits an dieser Stelle der Empfehlungen der DHS ist erkennbar, dass es sich nicht um innovative hochschultaugliche Vorstellungen handelt, sondern dass die bisherige Praxis der Therapieausbildungsinstitute (Ausbildung in therapeutischen Haltungen, Techniken und Methoden) nur unter das Dach von Hochschulen verlagert werden soll. Wahrlich keine Innovation für die Suchthilfe und die Hochschulen! 
In diesem Kontext besteht außerdem die Gefahr, dass die chronisch finanzschwachen deutschen Hochschulen vor dem Hintergrund der mit den neuen Abschlüssen gegebenen Möglichkeiten für die Interessen der alten Weiterbildungsträger („Neuer Wein in alten Schläuchen“) instrumentalisiert werden könnten. Auf jeden Fall bleibt zu fordern, dass suchtspezifische Studiengänge an deutschen Hochschulen ohne einen starken Forschungsbezug und auf der Basis solider Wissenschaftlichkeit nicht umgesetzt werden sollten. Ein suchtspezifischer Studiengang muss immer ein suchtwissenschaftlicher Studiengang sein und stellt von daher etwas anderes und im wissenschaftlichen Sinne mehr dar als eine suchttherapeutische Weiterbildung.

Erwähnenswert und kritikwürdig ist auch die Tatsache, dass bei der Entwicklung der Empfehlungen durch den DHS-Fachausschuss „Aus-, Fort- und Weiterbildung“ überhaupt nicht mit Vertretern der Hochschulen kommuniziert wurde. Inwieweit eine Abstimmung mit dem wissenschaftlichen Kuratorium der DHS erfolgt ist, das sich aus Vertretern der Hochschulen und Forschung zusammensetzt, ist dem Autor nicht bekannt.

Der Marktführer im Bereich für suchtwissenschaftliche Studiengänge, die Katholische Fachhochschule Nordrhein-Westfalen (KFH NW), wird im weiteren Verlauf des Papiers nur am Rande und als Beispiel (S. 6) erwähnt. Hier wäre im Vorfeld ein diskursiver Austausch möglich und wünschenswert gewesen. Üblichen akademischen Gepflogenheiten folgend melden wir uns deshalb nun mit dieser kritischen Stellungnahme zu Wort und möchten damit einen dringend nötigen Diskurs zum Thema eröffnen.

Abschließend heißt es in der Präambel, dass sich das Positionspapier „an Aufgaben, die sich aus den Arbeitsfeldern der Prävention, der sozialen Beratung, der niedrigschwelligen Arbeit bzw. dem Bereich der Überlebenshilfen und der Behandlung/medizinischen Rehabilitation der Suchthilfe ergeben“ (S. 3), orientiert. Diese Arbeitsfelder, deren Herleitung besser mit Bezug auf Suchtforschung einerseits und Arbeitsmarktbedeutung andererseits zu begründen wäre, decken mit Sicherheit nicht alle relevanten Tätigkeiten in der Suchthilfe ab: Was ist z.B. mit der Akutbehandlung, der qualifizierten Entgiftung, der Suchthilfe im Strafvollzug und in der Bewährungshilfe oder Suchthilfeangeboten in der Jugendhilfe? Aus den unzureichend hergeleiteten, aber sicherlich relevanten Arbeitsfeldern werden sodann Studienschwerpunkte (siehe Abschnitt 5: Aufbau von Masterstudiengängen in der Suchthilfe) abgeleitet, die wiederum ohne erkennbaren Bezug zu Forschung und Wissenschaft bleiben. Es wird lediglich erwähnt, dass Studienschwerpunkte entwickelt werden, „die gleichermaßen den Anforderungen des Berufsfeldes entsprechen und die Rahmenbedingungen von Praxis und Lehre berücksichtigen“ (S. 4). Es wird dadurch deutlich, dass die vorgelegten Empfehlungen vornehmlich dazu dienen, die Interessen verbandlich verankerter Weiterbildungen im Suchthilfebereich in die neue Struktur hochschulintegrierter Studiengänge retten zu wollen. Dabei bleibt natürlich unbenommen, dass sich hochschulexterne Träger der Weiterbildung Empfehlungen der hochschulexternen Weiterbildung geben können, was mit dem vorliegenden Papier wohl auch beabsichtigt ist. Insofern wird an dieser frühen Stelle in der Analyse der Empfehlungen deutlich, dass hier der unmögliche Versuch der Erstellung von Empfehlungskriterien für suchtspezifische Weiterbildungen innerhalb und außerhalb von Masterstudiengängen an wissenschaftlichen Hochschulen aus einem Guss („unter einem Dach“) versucht wird. Dies ist zweifelsohne kontraproduktiv und zum Scheitern verurteilt, da es kreative Innovationen verhindert, Flexibilität erschwert, die wissenschaftliche Orientierung der Hochschulen ignoriert und zur weiteren Verkrustung des bereits Verkrusteten führt.

\section{Strukturelle Entwicklung}

In einem zweiten Kapitel, strukturelle Entwicklung, der Empfehlungen wird die Entwicklung von Weiterbildungsansätzen in der Suchthilfe („tätigkeitsfeldspezifische Weiterbildungen“) in den letzten 30 Jahren skizziert. Dabei betonen die Autoren, dass es im Wesentlichen um die Adaption psychotherapeutischer Ansätze auf die Besonderheiten von Suchterkrankungen und Suchtdynamik ging. Dass bei der Rehabilitation Suchtkranker immer auch soziale Aspekte eine Rolle spielen, wird ausführlich erwähnt. Eher beiläufig werden die Empfehlungsvereinbarung Sucht vom 20.11.1978 und die Vereinbarung Abhängigkeitserkrankungen vom 1.7.2001 der deutschen Renten- und Krankenversicherungen erwähnt. Dass der Verband Deutscher Rentenversicherungen (VDR) mit diesen Vereinbarungen und den gänzlich unerwähnt bleibenden Beurteilungskriterien des VDR für Weiterbildungen in der Suchthilfe [6] bislang die eintscheidenden Anstöße geliefert hat, bleibt leider unerwähnt. Es ist lediglich die Rede von einer eindeutigen Forderung der Rentenversicherung „nach Professionalisierung und Spezialisierung für das Arbeitsgebiet Suchthilfe“ (S. 5).

Im weiteren Verlauf des Abschnitts „Strukturelle Entwicklung“ wird ausgeführt, dass mit „der Etablierung der Masterstudiengänge für Soziale Arbeit an deutschen Fachhochschulen ... seit 2001 ein Veränderungsprozess“( . 5) begann. Hier ist klarzustellen, dass seit 2001 zunächst postgraduale Weiterbildungsstudiengänge mit Abschluss Master of Arts oder Master of Science entstanden. Die konsekutiven (und vermutlich auch wenigen weiterbildenden) Masterstudiengänge für Soziale Arbeit werden im Wesentlichen erst ab dem Jahr 2007 etabliert werden. Darüber hinaus ist an dieser Stelle darauf hinzuweisen, dass ein suchtspezifischer Masterstudiengang eindeutig keinen Studiengang der Sozialen Arbeit (und auch keinen Studiengang der Medizin oder Psychologie) darstellen kann, sondern notwendigerweise ein interdisziplinärer Studiengang mit Studierenden aus verschiedenen Primärdisziplinen und Beiträgen aus höchst unterschiedlichen Wissenschaftsdisziplinen sein muss. Neben der Vernachlässigung der Wissenschaftsorientierung wird hier ein zweiter wesentlicher Schwachpunkt des DHS-Papiers deutlich: Es handelt sich fast durchweg um ein berufspolitisches Positionspapier aus der Perspektive der Sozialen Arbeit. Dies mag an geeigneter anderer Stelle seinen Platz haben, ist aber wohl weder im Auftrag und unter dem Label der DHS zu verantworten noch im Sinne der Adressaten und Nutznießer von suchtspezifischen Weiterbildungen zu akzeptieren. Der Fachausschuss „Aus-, Fortund Weiterbildung“, der sich überwiegend aus Fachvertretern der Sozialen Arbeit zusammensetzt, müsste hier entweder über den eigenen Schatten springen oder interdisziplinäres Knowhow integrieren.

Auch ein Arzt, der sich in einer Schwerpunktpraxis für Suchtmedizin auf die Arbeit mit Suchtkranken spezialisieren möchte, muss Gelegenheit zur Teilnahme an postgradualen Studiengän- 
gen der Suchthilfe im interdisziplinären Kontext haben. Gleiches gilt für einen niedergelassenen psychologischen Psychotherapeuten, der sich auf die Klientel der Suchtkranken und Suchtgefährdeten spezialisieren möchte. Vertreter dieser Berufsgruppen werden zwar nicht in großer Zahl an Masterstudiengängen teilnehmen, aber sie nicht zu bedenken oder gar auszuschließen ist völlig kontraproduktiv. Für zukunftsweisende akademische Weiterbildung im Bereich der Suchthilfe ist neben der Wissenschaftlichkeit die Interdisziplinarität ein unverzichtbares Qualitätskriterium - und zwar in Bezug auf die teilnehmenden Studierenden und die gelehrten Inhalte.

Abschließend heißt es dann im Abschnitt „Strukturelle Entwicklung“ zum Master-Trend: „Die Fachhochschul-Master (FH) stellen, mit akademischen Titeln versehen, eine Alternative zu den bisher außerhalb der Hochschulen am Weiterbildungsmarkt bestehenden Angeboten z. B. der Suchthilfe, dar. Beispielsweise hat sich die Katholische Fachhochschule in Nordrhein-Westfalen dieser Entwicklung angepasst und bietet gleichzeitig zur VDR anerkannten Zusatzqualifikation zum/zur Suchttherapeut/-in auch den postgradualen Studiengang mit Master-Abschluss für Suchtprävention und Suchttherapie an“ (S. 6). Abgesehen von der Tatsache, dass es im neuen Hochschulrecht keinen Master (FH), sondern nur einen Master-Abschluss (Master of Arts oder Master of Science) gibt, gleichgültig ob dieser an einer Universität oder Fachhochschule erworben wurde, verkennen die Autoren, dass der seit 2001 an der Katholischen Fachhochschule Nordrhein-Westfalen (KFH NW) in Köln etablierte postgraduale Masterstudiengang „Suchthilfe“ nicht irgendein Beispiel für die beschriebene Anpassungsleistung ist, sondern der erste und bislang immer noch einzige suchtwissenschaftliche Studiengang in Deutschland. Bei der Konzeption des Studiengangs [7] stand nicht irgendeine Anpassungsidee im Raum, sondern die Absicht, praktisches Handeln in der Suchthilfepraxis endlich erfolgreich mit den inzwischen umfangreich vorhandenen, aber meist von der Praxis nicht sehr stark rezipierten wissenschaftlichen Grundlagen der Suchthilfe in den verschiedenen Disziplinen fruchtbar zu verbinden. Der unlängst verstorbene Psychotherapieforscher Klaus Grawe formulierte die notwendige Haltung in vortrefflicher Weise noch kurz vor seinem Tod mit den Worten ${ }^{1}$, dass man dafür sorgen muss, dass Qualität in der Psychotherapie auf guten wissenschaftlichen und klinischen Füßen stehen muss. Dies gilt für die Suchttherapie, die auch eine fundierte Psychotherapie umfassen muss [8], in ebensolcher Weise. Diese Haltung liegt dem DHS-Empfehlungspapier leider nicht in erkennbarer Weise zugrunde.

Ein wissenschaftlich fundierter Studiengang zur Suchttherapie oder -prävention bedeutet qualitativ etwas völlig anderes als eine privatwirtschaftlich oder verbandlich getragene Therapieausbildung im Tätigkeitsfeld Sucht. Dies zu erkennen und die Konsequenzen daraus zu ziehen, wäre eine wichtige Aufgabe für den DHS-Fachausschuss „Aus-, Fort- und Weiterbildung“, wenn er sich mit suchtspezifischen Studiengängen an deutschen Hochschulen beschäftigen möchte.

\footnotetext{
1 Aus: „Ich bin kein Anhänger von Wahrheiten“, Gespräch mit Prof. Dr Klaus Grawe in: Report Psychologie, 2005, 30, 304-308.
}

Das zweite Kapitel der DHS-Empfehlungen endet dann mit dem Satz: „Es ist nur eine Frage der Zeit, wann weitere Institute in der deutschen Suchthilfe ihre bestehenden Abschlüsse diesen Standards anpassen werden. Alle Studiengänge in der Suchthilfe, welche die Belange der Suchthilfe relevant berücksichtigen (z.B. Medizin, Sozialpädagogik und Psychologie), sollten eine Abstimmung und Koordination mit den Fachverbänden und der DHS umsetzen“ (S. 6). An dieser Stelle wird ein weiterer wesentlicher Kritikpunkt an dem Papier deutlich: Neben dem bereits erwähnten Angriff auf die Freiheit von Lehre und Forschung und der Vernachlässigung suchtwissenschaftlicher Grundlagen für die Aus- und Weiterbildung in Suchthilfe wird deutlich, dass die Institute und Verbände einen Schwund ihres Einflusses und ihrer Bedeutung fürchten, wenn sich die Hochschulen zunehmend der Ausbildung in Suchthilfe annehmen würden. Daher rührt wohl die Vorstellung, dass diese sich mit der DHS und den Fachverbänden abstimmen sollten. Dass solche Abstimmungen sinnvoll und wichtig sein können, ist völlig unbestreitbar. Allerdings sollten sie dann - und hierfür ist das DHS-Papier leider ein schlechtes Vorbild - wechselseitig und partnerschaftlich erfolgen. Die Entwicklung und Akkreditierung des ersten Masterstudiengangs „Suchthilfe“ in Deutschland an der KFH NW geschah in einem engen interdisziplinären Abstimmungsprozess aus Praktikern, Verbandsvertretern und Wissenschaftlern. Schließlich geht es um eine Optimierung der Suchthilfepraxis, die am ehesten durch eine enge Kommunikation zwischen den Praxiseinrichtungen und den Hochschulen zu erreichen ist.

\section{Anforderungen an Masterstudiengänge in der Suchthilfe}

Das dritte Kapitel der DHS-Empfehlungen widmet sich den inhaltlichen Anforderungen an mögliche Masterstudiengänge in der Suchthilfe. Ausgehend von der Tatsache, dass die meisten Interessenten für derartige Studiengänge aus dem Bereich der Sozialen Arbeit kommen (werden), fokussiert das Papier auf die „Rahmenordnung für die Diplomprüfung im Studiengang Soziale Arbeit“, wie sie am 3. Juli 2001 von der Konferenz der Rektoren und Präsidenten der Hochschulen beschlossen worden ist. Die skizzierten Inhalte, die im Wesentlichen das Grundgerüst vieler FH-Diplomstudiengänge für die ehemaligen Fächer Sozialarbeit bzw. Sozialpädagogik (heute: Soziale Arbeit) umreißen, sind aus verschiedenen Gründen problematisch: Zum einen steht der grundständige Studiengang „Soziale Arbeit“ - und das wurde in der Präambel ausführlich erläutert - im Umbruch im Rahmen des Bologna-Prozesses, d.h. hin zur Veränderung in Bachelorund Masterstudiengänge. Insofern ist die nunmehr alte Rahmenstudienordnung für künftige, aufbauende Masterstudiengänge wenig verbindlich bzw. bereits erkennbar obsolet. Im Kapitel 3.1 des DHS-Papiers („Zur grundständigen Ausbildung der Sozialen Arbeit“) müsste also über die neuesten Entwicklungen im Rahmen von Bachelor-Studienordnungen in der Sozialen Arbeit berichtet werden. Diese haben zum Zeitpunkt der Abfassung der Empfehlungen auch schon vorgelegen (z.B. von Seiten des Fachbereichstages Soziale Arbeit). Zum anderen ist es - wie bereits angedeutet - fragwürdig, ob Masterstudiengänge in der Suchthilfe sich ausschließlich an grundständig ausgebildete Sozialarbeiter/Sozialpädagogen richten sollen. Auch wenn diese die Mehrheit in den Studiengängen (wie auch schon in den extramuralen Weiterbildungsgängen) ausmachen dürften, ist es im Sinne der Multi- und Interdisziplinarität in der Suchthilfe wenig begrüßenswert, dass überwiegend auf die Grundlagen nur einer Fach- 
disziplin rekurriert wird. Die Autorinnen und Autoren verkennen somit, dass Absolventen entsprechender Masterstudiengänge in der Suchthilfe interdisziplinär ausgebildete Fachkräfte darstellen müssen, und betrachten sie eher als Sozialarbeiter/Sozialpädagogen auf höherem Niveau („Master“). Es muss jedoch auf der Basis einer umfassenden „addiction science“ mit eigenständigen Forschungsthemen und -resultaten ein hochspezifisches neues Berufsprofil ausgebildet werden. Dies erfordert ein qualitatives und quantitatives Profil, das sich von dem in den DHS-Empfehlungen enthaltenen deutlich unterscheidet. Dort wird auf der Basis veralteter und monodisziplinärer Studienorientierungen aus der Sozialen Arbeit ein „Obersozialarbeiter“ mit einem MasterAbschluss skizziert.

Es braucht kaum mehr erwähnt zu werden, dass auch in diesem wichtigen Kapitel der Verweis auf die notwendigen wissenschaftlichen Grundlagen der Suchthilfe erneut völlig fehlt. Insofern ist der Bezug zur Rahmenstudienordnung „Soziale Arbeit“ von 2001 in einem weiteren Sinne zweifelhaft, als dass hierdurch die schlechte empirisch-wissenschaftliche Basisqualifikation der Absolventen des Faches Soziale Arbeit zum Leitbild späterer Master-Experten in der Suchthilfe transferiert wird. In gängigen suchtwissenschaftlichen Texten wird beklagt, dass eine Ursache für die mangelnde Beachtung wissenschaftlicher Erkenntnisse im Suchthilfebereich der zu geringe Anteil von Mitarbeitern in der Praxis mit einer wissenschaftlichen Ausbildung ist [9]. Da die Absolventen des Faches Soziale Arbeit nach wie vor die größte Berufsgruppe in der Drogentherapie und eine der wichtigsten in den anderen Suchttherapiefeldern darstellt [10], kann Bühringer [9] die Sozialarbeiter und Sozialpädagogen nicht als wissenschaftlich qualifiziertes Personal betrachtet haben. Dies steht natürlich in krassem Widerspruch zur Selbstdefinition des Faches, vertreten z. B. durch den Fachbereichstag Soziale Arbeit oder die Dekanekonferenzen in den verschiedenen Bundesländern. Im Kontext der DHS-Empfehlungen erfährt der Status quo der Sozialen Arbeit in Bezug auf Defizite in der wissenschaftlichen Grundausbildung stille Akzeptanz statt einer zukunftsfähigen Lösung.

Die im anschließenden Kapitel 3.2 berichteten Details zur Akkreditierung von Masterstudiengängen sind erneut fehlerhaft. So ist die Akkreditierungsagentur AHPGS (Freiburg) zwar auf Studiengänge im Sozial- und Gesundheitswesen spezialisiert, aber mitnichten für diese Studiengänge quasi bundesweit zuständig. Vielmehr gibt es bundesweit eine Reihe zuständiger und kompetenter Akkreditierungsagenturen (z. B. AQAS in Bonn), die solche Aufgaben in gleicher Weise zu übernehmen berechtigt und in der Lage sind. An dieser wie auch an vielen anderen Stellen der „Empfehlungen“ wird deutlich, dass dem DHS-Fachausschuss „Aus-, Fort- und Weiterbildung“ die Wissensgrundlagen für Abläufe und Regularien im Hochschulbereich offenbar fehlen. Mit entsprechender Beratung und Kommunikation - die aber für den Umkehrfall (siehe Kap. 2) angemahnt werden - wären die entsprechenden vielen sachlichen Fehler vermeidbar gewesen. Die Autoren schließen das dritte Kapitel wieder mit der Forderung nach enger Verzahnung des in der Weiterbildung erworbenen Wissens und Könnens mit den praktischen Anforderungen und Erfahrungen des Arbeitsalltags (S. 8) ab. Dies ist in der Tat richtig und wichtig, aber für akademische Studiengänge im Bereich der angewandten Wissenschaften nur eine Teilwahrheit. Insofern spiegelt der in den Empfehlungen folgende Satz das eingeschränkte Wissenschafts-Praxis-Verständnis des Fachausschusses wider. Dieser lautet: „Eine angemessene Qualifizierung können Masterstudiengänge nur leisten, wenn dieser enge Praxis-Ausbildungs-Transfer gesichert ist" (S. 8). Es ist zwingend zu fragen, wieso die Autoren den Forschungs-Praxis-Transfer völlig unerwähnt lassen. Die Frage ist an dieser Stelle nicht beantwortbar, macht aber deutlich, dass ein so wichtiges Gremium im Rahmen der DHS wie der Fachausschuss „Aus-, Fort- und Weiterbildung“, dessen Resultate von Entscheidungsträgern und Fachkräften gleichermaßen als wichtig angesehen werden dürften, auf der Basis schmalen wissenschafts- und hochschulbezogenen Wissens agiert.

\section{Arbeitsfelder}

Nach dem mit zwei Seiten eher knappen Kapitel zu den Anforderungen an Masterstudiengänge im Bereich der Suchthilfe folgt das mit sechs Seiten sehr umfänglich bemessene Kapitel zu den Arbeitsfeldern der Suchthilfe, auf die sich Masterstudiengänge beziehen sollten. An dieser Stelle kann auf diesen Abschnitt der Empfehlungen nur in gebotener Kürze eingegangen werden.

Als Arbeitsfelder der Suchthilfe werden genannt:

a) Prävention,

b) soziale Beratung,

c) niedrigschwellige Arbeit bzw. Überlebenshilfen,

d) Behandlung bzw. medizinische Rehabilitation und

e) Tätigkeiten im Bereich der Selbsthilfe.

Zunächst ist kritisch zu fragen, wie umfassend die genannten Arbeitsfelder jeweils sind und welche Interaktionen und Überschnittsmengen zwischen den Arbeitsfeldern bestehen. Stellen die genannten Bereiche tatsächlich Arbeitsfelder in einem eher groben, umfassenden Sinne dar, so ist verstehbar, dass suchtbezogene Tätigkeitsfelder (eher „molekular“, also differenzierter) wie z.B. Dokumentation, Bewährungs- und Straffälligenhilfe, Wohnungslosenhilfe hier unerwähnt bleiben. Auf der anderen Seite ist dann die Aufgliederung der Arbeitsfelder in unterschiedlich große Bereiche hinsichtlich der dort Tätigen und Beschäftigten auffällig. So sind die Tätigkeitsfelder Beratung und Behandlung im Suchthilfebereich äußerst umfänglich. Auch das Tätigkeitsfeld Prävention gewinnt immer mehr an Bedeutung. Der Bereich der Überlebenshilfen ist in den deutschen Großstädten sehr gut ausgebaut, in anderen Regionen so gut wie nicht vorhanden und somit ein weniger umfangreicher Arbeitssektor. Das Stichwort „Tätigkeiten im Bereich der Selbsthilfe" bleibt unscharf und erscheint unter arbeitsmarktpolitischen Aspekten kaum bedeutsam. Dass Selbsthilfe als eigenes professionelles Arbeitsfeld auftaucht, ist kaum verständlich und widerspricht wohl auch dem Grundgedanken der Selbsthilfe. Damit kann lediglich die Kooperation und Vernetzung zwischen Fachkräften und Suchtselbsthilfe gemeint sein. Diese Tätigkeiten als gleichberechtigt neben die bereits genannten zu setzen, ist weder aus fachlicher noch aus arbeitsmarktpolitischer Sicht zu rechtfertigen. Vielmehr sollten diese Tätigkeiten, zusammen mit den vielfältigen Aufgaben der Nachsorge, Erwähnung finden. Dabei könnte auf einen eigenständigen „Cluster“ verzichtet und die entsprechenden Aufgaben dem Bereich Behandlung zugeordnet werden.

Schließlich ist der in den Empfehlungen benutzte Terminus der „sozialen Beratung“ zu hinterfragen. Dieser ist weder eindeutig 
definiert noch hinreichend etabliert, um die Tätigkeiten in der Beratung Suchtkranker zu beschreiben. Erneut ist eher zu vermuten, dass hier berufsständische Politik betrieben werden soll, da dieser Begriff (ähnlich wie soziale Gruppenarbeit) von der Sozialen Arbeit als Proprium reklamiert werden dürfte. In der Praxis geht es um viel mehr, wie auch die von den Autoren vorgenommene Aufzählung der Inhalte der Beratungen für Suchtkranke (S. 11) verdeutlicht. Hier sind u.a. die Bearbeitung von Problemen und die Vermittlung von Hilfen, körperliche, soziale und seelische Folgeschäden, ambivalente motivationale Veränderungsbereitschaft, psychosoziale Konfliktlagen der Angehörigen sowie Präventionsarbeit und -dienstleistungen genannt. Dieses Arbeitsfeld wird gemeinhin mit dem Begriff der psychosozialen Beratung bezeichnet und es ist nicht ersichtlich, aus welchem Grund dieser Begriff in den Empfehlungen nicht verwendet wird.

Insgesamt wäre es wünschenswert, die in der Suchthilfe dominierenden Arbeitsfelder und die zugehörigen Tätigkeiten differenzierter und mit Bezug sowohl zum Arbeitsmarkt als auch zur bislang geleisteten wissenschaftlichen Fundierung aufzulisten (vgl. [10]). Dann würde deutlich, dass Tätigkeiten in den Bereichen Leitung, Führung, Dokumentation, Berichterstattung, Öffentlichkeitsarbeit, Konzeptentwicklung, Innovation, Moderation und Vernetzung, die im vorliegenden Papier völlig unerwähnt geblieben sind, in der Praxis eine große und wichtige Bedeutung haben. Außerdem ist das Schnittstellenmanagement mit benachbarten Arbeitsfeldern (z. B. Jugendhilfe, Psychiatrie, Wohnungslosenhilfe, Straffälligen- und Bewährungshilfe usw.) von enormer Relevanz und in entsprechenden Masterstudiengängen unbedingt zu berücksichtigen.

\section{Aufbau von Masterstudiengängen in der Suchthilfe}

In diesem mit knapp sieben Seiten längsten Kapitel der Empfehlungen werden die Konsequenzen aus den vorangegangenen Ausführungen gezogen. Zunächst heißt es: „Ein Masterstudiengang in der Suchthilfe sollte die unmittelbare Verzahnung von wissenschaftlich fundiertem Studium und suchtspezifischem Praxisbezug“ (S. 15) darstellen. Es handelt sich um die einzige Passage in den gesamten Ausführungen, die einen Bezug zur Wissenschaft herstellt. Leider wird dies in den folgenden Ausführungen quasi vergessen und in keiner Weise mehr aufgegriffen. Dabei wäre es gerade Aufgabe eines solchen Papiers, aufzuzeigen, wie die geforderte Verzahnung in der Studienpraxis denn geschehen soll. Zunächst folgt auf Seite 15 die Fehlinformation, dass Masterstudiengänge an Universitäten die Möglichkeit der Promotion eröffnen. Richtig ist vielmehr, dass bei Masterabschlüssen nicht zwischen Universitäten und Fachhochschulen unterschieden wird und daher allen Absolventen mit einem Master-Titel grundsätzlich die Promotion offen steht.

Die Autoren sehen eine Zweiteilung der Studiengänge vor. Im ersten Teil (die Rede ist von zwei Basissemestern) soll Grundlagenwissen zu Suchtfragen vermittelt werden. Hierzu zählen fachlich-inhaltliche und personale Kompetenzen in der Suchthilfe, sucht- und drogenspezifisches Basiswissen, Beratung, Betreuung und Behandlung, suchtrelevante Akteure, Einrichtungen und Hilfesysteme, politische und rechtliche Rahmenbedingungen, Zielgruppenorientierung, Projektarbeit sowie Qualitätsmanagement und -sicherung. Die Aufzählung mutet eher wie eine
Sammlung von Stichwörtern an und lässt wenig curriculare Logik erkennen. Es fehlt leider der klare Bezug zu Grundlagenwissenschaften, wie z.B. Suchtmedizin und Suchtpsychologie, mit entsprechenden Kategorisierungen. Stattdessen werden unter „suchtrelevante Akteure, Einrichtungen und Hilfesysteme“ u.a. Case-Management und Schnittstellenmanagement erwähnt. Ein Bezug zu wissenschaftlichen Ansätzen, z. B. im Bereich Versorgungsforschung, Soziologie der Hilfesysteme, soziologische Analyse von Zugangsschwellen und Gesundheitsökonomie der Suchthilfe, unterbleibt völlig. Ähnlich werden im Modul „Zielgruppenorientierung“ u.a. die gemeinsame Erarbeitung von Zielen mit Klienten sowie die Techniken zur Gesprächsführung gelehrt. Dies sind erkennbar aber Kompetenzen, die eher in den zweiten Teil des Studiums - von den Autoren Hauptstudium oder Praxis genannt - gehören. Abgesehen davon, dass in den künftigen Bachelor- und Masterstudiengängen nicht zwischen Vor- und Hauptstudium unterschieden wird, bleibt die Zuordnung vieler Elemente aus den „Basissemestern“ unklar und nicht legitimiert.

Im zweiten Studienteil ist eine Fokussierung auf bestimmte Praxisthemen und -felder vorgesehen: „Zur weiteren Spezialisierung bzw. arbeitsfeldorientierten Vertiefung mit einem Theorieund Praxisteil“" (S. 15) wählen die Studierenden drei von insgesamt fünf Studienschwerpunkten aus. Hierfür werden die im vorausgegangenen Kapitel „Arbeitsfelder“ benannten fünf Bereiche wiederum angeboten. Nunmehr stehen also die Bereiche Beratung, Behandlung, Prävention, Überlebenshilfen und Selbsthilfe quasi gleichberechtigt nebeneinander. Hier dürfte weniger mehr sein:

Studienschwerpunkte sollten sinnvollerweise nur solche Tätigkeitsfelder sein, die einerseits ausreichende bis gute Arbeitsmarktchancen bieten (z.B. Suchttherapie) und die andererseits bereits über ein mindestens erkennbares Maß an wissenschaftlicher Profilierung und Elaboration (einschließlich Methodenentwicklung) verfügen. Ob diese Kriterien bei den „Studienschwerpunkten“ Förderung der Selbsthilfe und Überlebenshilfen ausreichend erfüllt sind, darf bezweifelt werden.

Außerdem sollten Master-Studierende im Sinne einer wirklichen Qualifizierung nicht dazu angeleitet werden, eine zu große Zahl von Schwerpunkten zu studieren (das DHS-Papier schlägt jeweils 3 aus 5 vor). Es ist nicht ersichtlich, wieso die Studierenden gerade drei dieser Felder auswählen sollen. Dies hätte die Gefahr, dass das erworbene Wissen rudimentär und oberflächlich bleibt und daher letzten Endes nicht praxistauglich ist.

Insgesamt besteht beim DHS-Papier die Gefahr, dass Studienschwerpunkte mit verschiedenartiger Reichweite und Qualität als gleichwertig mit anderen in ein Normkorsett gezwungen werden. Im Sinne eines wissenschaftlich fundierten und arbeitsmarktgerechten Studiengangs kann vom Studium vieler kleiner Schwerpunkte nur abgeraten werden. Dies widerspricht im Übrigen natürlich auch der Wortbedeutung eines „Schwerpunkts“. Daran ändert auch die Vorgabe der Auswahl von drei aus fünf Schwerpunkten nichts.

Besser und geeigneter wäre hier eine andere Aufteilung („Zuschnitt“) der Studienschwerpunkte, z. B. in Prävention, Beratung, 
Behandlung, Managementaufgaben, mit einer Fokussierung auf zwei Bereiche. Der Bereich Managementaufgaben könnte dann auch die Kooperation und Kommunikation mit der Suchtselbsthilfe sowie Öffentlichkeitsarbeit umfassen. Überlebenshilfen und Krisenintervention könnten thematisch dem Bereich „Behandlung" zugeordnet werden. Auch ist an dieser Stelle wiederum eine durchgängige stärkere Berücksichtigung der wissenschaftlichen Grundlagen der Suchthilfe und der Ansätze der angewandten Suchtforschung anzumahnen.

\section{Zusammenfassung}

Das abschließende sechste Kapitel der Empfehlungen fasst insbesondere die im vorausgegangenen Abschnitt aufgelisteten Studienschwerpunkte zusammen. Hier wird nochmals versucht, die Berücksichtigung des „Arbeitsfeldes“ Selbsthilfe zu legitimieren: „Der Bereich der Selbsthilfe bietet trotz des überwiegend ehrenamtlichen Engagements ebenfalls ein Arbeitsfeld für Sozialarbeiter/-innen und Sozialpädagoginnen“ (S. 22). Leider werden erhärtende Zahlen vom Arbeitsmarkt dieser bloßen Behauptung nicht beigefügt. Möglicherweise spielen hierbei das Proporzdenken und die politische Korrektheit innerhalb des DHS-Fachausschusses eine zu große Rolle. Leider unterbleibt eine kritische Würdigung der Empfehlungen am Ende des Papiers, so dass dies im Folgeabschnitt zu leisten ist.

\section{Kritische Würdigung der DHS-Empfehlungen}

Die wichtigsten Kritikpunkte wurden im Rahmen der Analyse der einzelnen Abschnitte bereits erwähnt. Sie werden am Ende nochmals pointiert zusammengefasst:

1. Die DHS-Empfehlungen skizzieren einen unwissenschaftlichen Studiengang: Die Empfehlungen stellen keinen geeigneten Bezug zur Suchtforschung her, vernachlässigen die Forschungsperspektive völlig und versuchen unzulässigerweise praxeologische Weiterbildungskonzepte an wissenschaftliche Hochschulen zu transferieren. Die Reichweite des DHS-Papiers, so wie es im Moment vorliegt, kann sich eindeutig nur auf Weiterbildungsgänge außerhalb von Hochschulen beziehen. Eine Integration dieser Weiterbildungsgänge in den Hochschulbereich sollte im Übrigen auch nur auf der Basis eines Diskurses mit Hochschulvertretern geschehen. Dass dies bislang von Seiten des DHS-Fachausschusses unterblieben ist, ist nicht nur bedauerlich, sondern ein Kunstfehler im Sinne curricularer Entwicklungsmethodik.

2. Die DHS-Empfehlungen stellen berufsständische Politik im Gewand von Hochschulcurricula dar: In den Empfehlungen wird ein monodisziplinärer Blick aus der Perspektive der Sozialen Arbeit immer wieder deutlich. Da aber schon die VDR-Vereinbarung ausdrücklich auch im Bereich der Weiterbildung im Tätigkeitsfeldbereich der Suchthilfe Interdisziplinarität fordert, ist eine derartige monodisziplinäre Haltung in keiner Weise akzeptabel. Die DHS, die hier die Verantwortung für ihren Fachausschuss „Aus-, Fort- und Weiterbildung“ trägt, sollte für Interdisziplinarität in ihren Ausschüssen sorgen.

3. „Alter Wein in neuen Schläuchen“: Bedingt durch die beiden ersten Kritikpunkte ergibt sich der dritte. Als Resultat wird nämlich nichts anderes propagiert, als die bisherigen hochschulexternen Curricula nunmehr im Kontext der Hochschu- len durchzuführen. Den im Fachausschuss „Aus-, Fort- und Weiterbildung“ vertretenen Verbänden geht es offenbar primär um den Erhalt der bisherigen Weiterbildungspraxis und der damit verbundenen Strukturen. Die „Empfehlungen“ lassen durch die Perpetuierung der alten Weiterbildungskonzepte erkennen, dass es primär um den Erhalt von Einfluss und Ressourcen und nicht um Innovation und Modernisierung der Suchthilfe geht, was dringend angezeigt wäre.

Ein Bezug zum wissenschaftlichen Denken, das in der Suchthilfe immer wichtiger wird und nicht mehr entbehrt werden kann, fehlt in den Empfehlungen fast völlig. Dass Studiengänge an wissenschaftlichen Hochschulen - im Master-Bereich - deutlich stärker als in grundständigen Bachelorstudiengängen für wissenschaftliche Analyse- und Reflexionsfähigkeiten qualifizieren müssen, bleibt völlig unerwähnt. Eine Vertiefung in empirische Forschungsmethoden - ohnehin die Schwachstelle vieler FHStudiengänge zur Sozialen Arbeit in der Konkurrenz mit Absolventen aus Medizin, Psychologie und Soziologie - ist nicht vorgesehen.

Ein wissenschaftliches Studium im Bereich der Suchthilfe muss alleine schon aus arbeitsmarktpolitischen Gründen dem Bereich der medizinischen Rehabilitation (ambulante und stationäre „Suchttherapie“) sehr großen Raum widmen, da hier die meisten Stellen vorhanden sind und auch in Zukunft zu besetzen sein werden. Welche beruflichen Chancen (Anstellungschancen) haben Experten der Suchthilfe in den Tätigkeitsfeldern „Förderung der Selbsthilfe"? Auch ein eher unspezifisch wirkender Tätigkeitsbereich „Soziale Beratung“ wird keine ausreichende Arbeitsmarktnachfrage erleben, wenn er sich nicht ganz eindeutig auf die ambulante Behandlung und Rehabilitation Suchtkranker und -gefährdeter bezieht. Und dies umfasst mehr als den berufsständisch - im Sinne der Sozialen Arbeit - definierten Begriff der „Sozialen Beratung“.

Man kann allen Hochschulen, die sich ernsthaft um die Entwicklung und Etablierung von Studiengängen im Bereich der Suchthilfe bemühen möchten, und anderen Entscheidungsträgern nur dringend abraten, sich an den Empfehlungen der DHS hierfür zu orientieren. Folgen sie diesen Empfehlungen, entwickeln sie Studiengänge, die nichts anderes sind als die bisher von den Verbänden angebotenen suchtspezifischen Therapieweiterbildungen mit dem äußeren Etikett „Hochschule“. Solche Studiengänge dürfen niemals als qualitativ hochwertige Studiengänge akkreditiert werden. Sie würden die Kultur des oberflächlichen, unkritischen und unwissenschaftlichen Handelns in der Suchthilfe noch mehr vertiefen. Das Gegenteil ist notwendig und die Praxis hat es verdient.

\section{Literatur}

${ }^{1}$ Deutsche Hauptstelle für Suchtfragen DHS (Hrsg). Empfehlungen zu postgraduierten Curricula in der Suchhilfe. http://www.dhs-intern.de/pdf/Empfehlungen-postgraduierten-Curricula.pdf (20.8.2005).

${ }^{2}$ Bossong H. Wenn soziale Arbeit Zukunft haben soll: Kritische Überlegungen zu Modernität und Professionalität in der ambulanten Suchtkrankenhilfe in Deutschland. Sucht aktuell 1996; 3 (1/2): 4-7 
${ }^{3}$ Klein M. Tradition, Statuts quo und Innovation in der stationären Suchttherapie - Betrachtungen zur Geschichte der Suchthilfe und zukünftigen Entwicklungsaufgaben. In: Fachverband Sucht e.V (Hrsg). Suchttherapie unter Kostendruck. Beiträge des 10. Heidelberger Kongresses 1997. Schriftenreihe des Fachverbandes Sucht e.V. 21. Geesthacht: Neuland, 1998: 107-119

${ }^{4}$ Körkel J. Denkstile über Sucht: Beginn eines Wandels. In: Fachausschuss Soziotherapie des Wissenschaftsrates der AHG (Hrsg). Entweder oder sowohl als auch? Aspekte der Soziotherapie Alkoholabhängiger. Verhaltensmedizin heute, Fortschritte in der Rehabilitation, Heft 5. Hilden: Allgemeine Hospitalgesellschaft mbH, 1995: 8-17

${ }^{5}$ Krausz M. Nichts ist so beständig wie der Wandel - Anforderungen an das Suchthilfesystem in den nächsten Jahren. In: Krausz M, Haasen C (Hrsg). Kompendium Sucht. Stuttgart: Thieme, 2004: 153-156

${ }^{6}$ Ammer F, von Boetticher I, Kulick B et al. Beurteilung von Weiterbildungen für Einzel- und Gruppentherapeuten (Tätigkeitsfeld Sucht) gemäß der Anlage 1 der Empfehlungsvereinbarung vom 20. November 1978 und $\S 5$ Abs. 4 der Empfehlungsvereinbarung Ambulante Rehabilitation Sucht vom 29. Januar 1991. Deutsche Rentenversicherung 1992; 7-8: $468-473$

${ }^{7}$ Klein M, Hoff T. Evaluation des postgradualen Studiengangs ,Master of Science (M.Sc.) in Addiction Prevention and Treatment' - Suchthilfe als Studiengang zur Verbesserung der therapeutischen und wissenschaftlichen Kompetenz von Suchthilfemitarbeitern. Suchttherapie 2004; 5: 30-36

${ }^{8}$ Klein M. Die Bedeutung der Psychotherapie für die Drogenhilfe. In: Arbeitsgemeinschaft Drogenarbeit und Drogenpolitik in NRW e.V (Hrsg). Drogenhilfe: Sozialarbeit, Psychotherapie und Medizin. Der Stellenwert der verschiedenen Professionen. Tagungsbericht 2000. Niederkrüchten: Arbeitsgemeinschaft Drogenarbeit und Drogenpolitik in NRW e.V, 2001: 6-13

${ }^{9}$ Bühringer G. Suchtkrankenhilfe und Forschung in Deutschland: eine problematische Beziehung. In: Deutsche Hauptstelle gegen die Suchtgefahren (Hrsg). Suchtkrankenhilfe in Deutschland. Geschichte Strukturen - Perspektiven. Freiburg: Lambertus, 1997: 259-269

${ }^{10}$ Klein M. Praxisfeld Suchthilfe. In: Badry E, Buchka M, Knapp R (Hrsg). Pädagogik. Grundlagen und Arbeitsfelder. 4., überarbeitete Auflage. Neuwied: Luchterhand, 2003: 481 -494

\section{Erratum}

\section{Neuenschwander, U. Frick, G. Gmel, J. Rehm. Prävalenz und Determinanten für Risikokonsum. Suchttherapie 2005; 6: $126-132$}

Leider wurde eine falsche Abbildung gedruckt. Nachstehend die richtige.

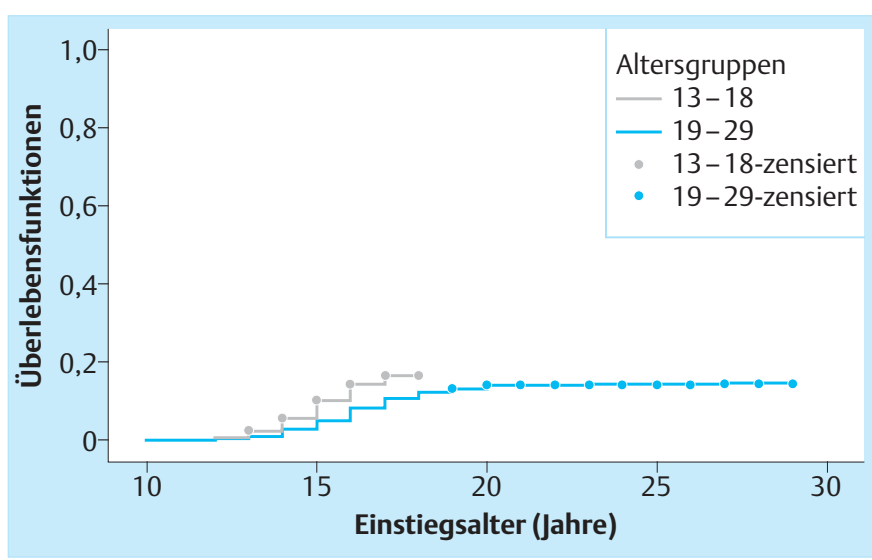

Abb. 1 Kumulativ-komplementäre Überlebensfunktionen für das Einstiegsalter nach Altersgruppen (Log-Rank-Wert = 23,2, p = 0,000). 